\title{
The Mediating Role of the Environment in Explaining Participation of Children and Youth With and Without Disabilities Across Home, School, and Community
}

\author{
Dana Anaby, PhD, ${ }^{a}$ Mary Law, PhD, ${ }^{b, c}$ Wendy Coster, $\mathrm{PhD}^{\mathrm{d}}$ Gary Bedell, PhD, ${ }^{\mathrm{e}}$ \\ Mary Khetani, ScD, ${ }^{f}$ Lisa Avery, MSc, ${ }^{g}$ Rachel Teplicky, MSc $^{c}$
}

From the ${ }^{a}$ School of Physical and Occupational Therapy, McGill University, Montreal, Quebec, Canada; ${ }^{b}$ School of Rehabilitation Science and ${ }^{c}$ CanChild Centre for Childhood Disability Research, McMaster University, Hamilton, Ontario, Canada; ${ }^{d}$ Department of Occupational Therapy, Sargent College of Rehabilitation Sciences, Boston University, Boston, MA; ${ }^{e}$ Department of Occupational Therapy, Tufts University, Medford, MA; ${ }^{f}$ Department of Occupational Therapy, Colorado State University, Fort Collins, CO; and ${ }^{g}$ Avery Information Services Ltd, Orilla, Ontario, Canada.

\begin{abstract}
Objective: To test the effect of personal and environmental factors on children's participation across 3 different settings (home, school, community); to ascertain the interrelations between these factors; and to propose and test 3 models, 1 for each setting, using structural equation modeling.

Design: Survey, cross-sectional study, and model testing.

Setting: Web-based measures were completed by parents residing in North America in their home/community.

Participants: Parents $(\mathrm{N}=576)$ of children and youth with and without disabilities, $(\mathrm{n}=282 \mathrm{and} n=294$, respectively), ages 5 to 17 years (mean age, 11y 2mo), completed the Participation and Environment Measure for Children and Youth (PEM-CY).

Interventions: Not applicable.

Main Outcome Measures: The PEM-CY measured levels of participation frequency and involvement, as well as environmental barriers and supports of participation, in each of the following 3 settings: home, school, and community. Information about the child's health condition and functional issues was also collected.

Results: All 3 models fit the data well (comparative fit index, .89-.97) and explained 50\% to 64\% of the variance of participation frequency and involvement. Environmental barriers and supports served as significant mediators between child/personal factors (income, health condition, functional issues) and participation outcomes, across all models. The effect of the environment was most pronounced, however, in the community setting.

Conclusions: Our findings highlight the unique role of the environment in explaining children's participation across different settings and, therefore, support the development of interventions targeting modifiable environmental factors.
\end{abstract}

Archives of Physical Medicine and Rehabilitation 2014;95:908-17

(C) 2014 by the American Congress of Rehabilitation Medicine

Participation in home, school, and community activities has a positive impact on children's health, development, and wellbeing. ${ }^{1,2}$ Through participation, children acquire skills, achieve

Supported by the U.S. Department of Education, National Institute on Disability and Rehabilitation Research (grant no. H133G070140).

No commercial party having a direct financial interest in the results of the research supporting this article has conferred or will confer a benefit on the authors or on any organization with which the authors are associated. physical and mental health, and develop social networks that are crucial for their transition to adulthood. ${ }^{3}$ It is important, therefore, to develop knowledge about activity patterns of children and youth, as well as the factors that impact these patterns. Environmental factors are of particular importance because they are potentially modifiable.

The participation of children and youth with disabilities, however, is restricted in comparison with their typically developing peers. ${ }^{4-8}$ Striking differences were found in a sample, 
reanalyzed in this study, of 576 children and youth living in Canada and the United States; $37 \%$ of children and youth with disabilities never took part in organized physical activities in the community, as compared with only $10 \%$ among their typically developing peers. ${ }^{9}$ In Europe, a large comparative study ${ }^{10}$ of more than 800 children with cerebral palsy and the general population $(n=2939)$ revealed similar discrepancies.

Participation is a complex concept that is influenced by personal factors related to the child and family and also by environmental factors. ${ }^{11}$ Prior research indicates that participation of all children is influenced significantly by age, ${ }^{12-15}$ sex,,${ }^{5,10,13,16}$ and income. ${ }^{13}$ Among children with disabilities, the severity of their condition, ${ }^{17}$ as well as their functional abilities, ${ }^{18}$ is also known to influence changes in participation over time. With accumulating knowledge, both theoretical ${ }^{11}$ and empirical, ${ }^{19}$ it is clear that the environment is inextricably linked to participation. A recent scoping review ${ }^{20}$ indicated that all aspects of the environment identified by the International Classification of Functioning, Disability and Health (eg, physical accessibility, services and programs, attitudes) served as a barrier, as a support, or both, for participation of children and youth with various types of disabilities. The most common facilitators involved the social support of family and friends and geographic location. The most common barriers included attitudes, physical environment, transportation, policies, and lack of support from staff and service providers. Another qualitative meta-synthesis ${ }^{21}$ further supports the impact of the environment on participation among youth with disabilities.

The specific role that the environment plays in the presence of other factors is not well understood. Studies imply that the environment has a direct impact on participation, as levels of participation varied across 8 European districts/countries, ${ }^{22}$ and these regions differ in terms of their accessibility. ${ }^{23}$ Another study, ${ }^{24}$ however, tested a complex model for predicting participation and found that the environment had an indirect effect on participation through its effect on the child's abilities. To date, the environment has not been directly measured as part of the assessment of participation, and in order to disentangle this relationship, other approaches are required. The Participation and Environment Measure for Children and Youth (PEM-CY) ${ }^{25}$ is an innovative, comprehensive, psychometrically sound measure that links participation to the environment across different settings (home, school, community) and serves as a promising tool to capture specific aspects of the environment that impact participation.

The purpose of this study is to examine factors that affect participation across home, school, and community settings and to reveal how these factors relate to one another. The proposed model describes our overall hypothesis of the study (fig 1) and is based on previous conceptual and empirical work. ${ }^{11,24}$ Overall, the model illustrates that the environment mediates the relationship between the complexity of a child's condition (disability, health

\section{List of abbreviations:}

CFI comparative fit index

CI confidence interval

HC health condition

PEM-CY Participation and Environment Measure for Children and Youth

RMSEA root mean square error of approximation SEM structural equation modeling
}

condition, functional issues) and participation (ie, frequency and involvement). Previous analysis of PEM-CY data, ${ }^{26}$ along with findings of other studies, ${ }^{24}$ indicated that sex had a negligible effect on participation in the presence of other factors and was therefore excluded from this model.

\section{Methods}

\section{Participants and procedures}

Parents were recruited through community-based organizations and service groups for children and families in Canada and the United States (see appendix 1 for recruitment strategies). Eligible participants had to be parents or legal guardians of a child aged 5 to 17 years, with or without a disability, and able to read English. Parents were directed via a weblink to a secure website to provide informed consent and to complete the demographic questionnaire, followed by the PEM-CY. Parents were asked to complete these questionnaires in a single sitting, which is a potential respondent burden and may explain the missing data in some cases. The ethics board of each of the participating universities approved the study.

\section{Measures}

Participation frequency and involvement as well as environmental factors were measured using the PEM-CY. This parent-report assessment includes 25 sets of activities across 3 different settings: home (10 items), school (5 items), and community (10 items). For each setting, environmental aspects that impact the child's participation (eg, resources, attitudes, availability of programs, accessibility) are assessed separately: 13 environment-related items for home, 17 for school, and 16 for community. Parents indicated whether each item was an environmental barrier, a facilitator, or both, to their child's participation. Scales and scores of the PEM-CY are described in table 1. The PEM-CY has demonstrated sufficient reliability (internal consistency, .59-.91; test-retest reliability, .58-.95) and was able to distinguish between children with and without disabilities across all scales (effect size, $.51-1.44$ ), supporting its validity. ${ }^{25}$

A demographic questionnaire was completed by parents to gather information about child/family contexts, including whether their child has a disability and which of a list of 11 functional issues affected their child's daily functioning (eg, communication with others, moving around). Parents also reported specific conditions of the child using a list of 13 health conditions (eg, intellectual delay, attention-deficit disorder). A mean number of health conditions as well as a mean number of functional issues were calculated to represent the complexity of the condition.

\section{Data analysis}

This study is a comprehensive secondary analysis of data published elsewhere. ${ }^{9}$ Initial examination of the distribution of the observed factors/indicators in each of the models was performed to assess assumptions of normality by using multivariate kurtosis; a result $>1.96$ indicated nonnormality. In addition, patterns of missing data were examined. Structure equation modeling (SEM) analysis was used to test the structural model of each setting (home, school, community), resulting in 3 models while accounting for the constructs of the latent variables of participation: frequency and 


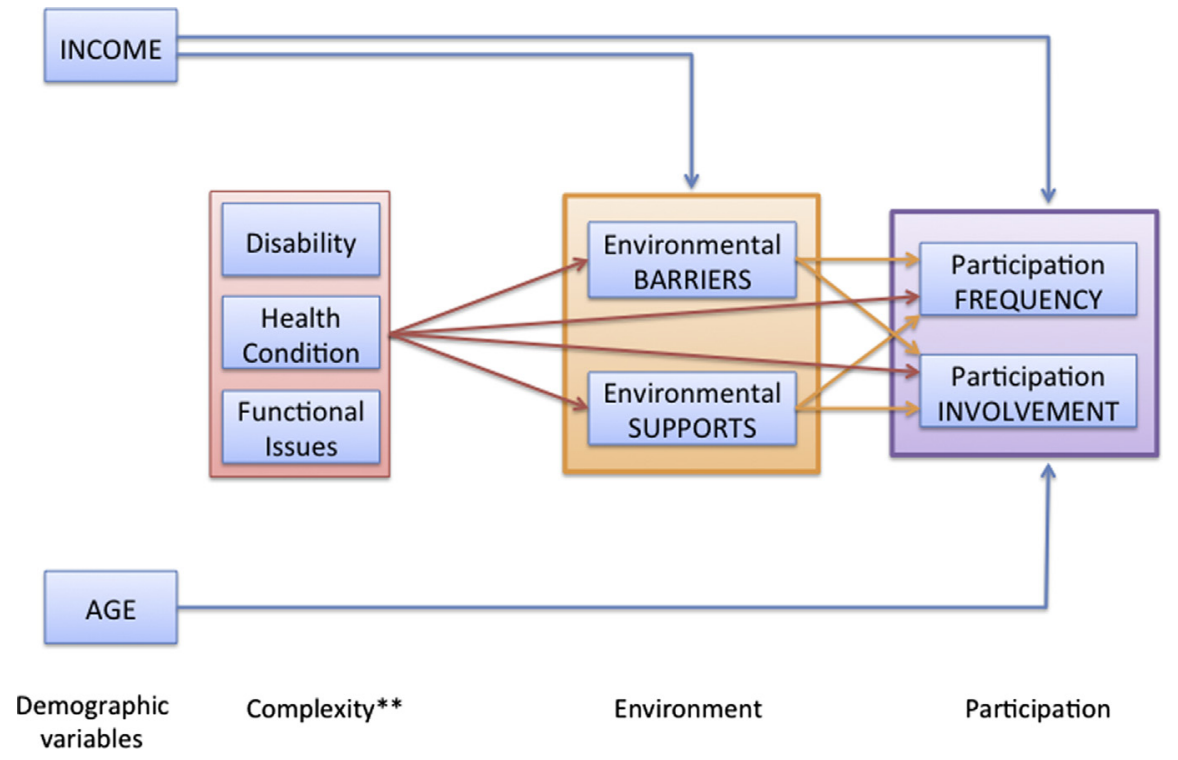

Fig 1 The proposed model. Both income and age serve as exogenous variables in which income has a direct effect on both environmental barriers/ supports and participation. Other exogenous variables representing the complexity of a child's condition in terms of presence of a disability and the number of functional issues and health conditions, have a direct effect on participation (ie, frequency and involvement) in each setting (home, school, community) and an indirect effect on participation through environmental barriers and supports. **Complexity of child's condition.

involvement (10 indicators/items for home, 5 for school, 10 for community). Three separate models were analyzed because of the conceptual foundation of the PEM-CY, ${ }^{27}$ where settings are distinct and variables are unique to each setting (eg, barriers in the community is a different variable than barriers in the home).

Each model was fit using a structural means model in combination with full-information maximum likelihood, to minimize the effects of missing data and lack of normality on the results. The common assumption that data are missing at random was made. Two indices of fit were used to evaluate the appropriateness of the models: the comparative fit index (CFI) and the root mean square error of approximation (RMSEA). The cutoff criteria for accepting the model was $\mathrm{CFI} \geq .90$ and RMSEA $<.06$ to $.08 .^{28}$ These indices have been shown to be free of sampling bias and to account for model complexity. Robust statistics, in the form of Yuan-Bentler statistics (ie, CFI corrected), were also used to account for nonnormality and missing data. When a relatively high proportion of cases with some missing data was observed, a second model was tested using listwise deletion of missing data, and parameters and indices of fit were then compared (appendix 2). Standardized estimates of the paths and the overall $R^{2}$ were reported to evaluate the extent to which the model can explain the outcomes of participation. Analyses were undertaken using EQS 6.2. ${ }^{\text {a }}$

Based on the rule of thumb recommended by Bentler and $\mathrm{Chou}^{29}$ for sample size (ie, a minimum of 5 cases per parameter estimate in the model and a minimum of 15 cases per measured variable), our study sample of 576 parents had more than the minimum required to test the model using SEM.

\section{Results}

\section{Participant characteristics}

The PEM-CY was completed by 282 parents of children with disabilities and 294 parents of typically developing children $(\mathrm{n}=576)$ ages 5 to 17 years, where most of the children were boys (54\%). Most families were living in urban/suburban areas $(80 \%)$ and had higher-than-average household incomes $(62 \%$ earned $>\$ 80,000 / y)$. The most commonly reported health conditions included orthopedic impairment (36\%), developmental delay $(26 \%)$, and speech-language disorders (21\%). The functional issue reported by the highest percentage of parents was attention $(80.7 \%)$, followed by memory and learning (73\%) (see Coster et $\mathrm{al}^{25}$ for detailed information about the diagnostic conditions and type of functional limitations). Table 2 describes the sample characteristics.

\section{Home model}

There were 576 cases available for analysis in the home setting including 109 cases with some missing data. The normalized estimate of multivariate kurtosis was 38.5 , indicating that the data were not multivariate normal. The factorial structure of the 2 latent variables in the model - that is, participation frequency and participation involvement-was confirmed. All of the frequency items (standardized loadings range, .19-.73) and involvement items (standardized loadings range, .26-.76) were significant indicators of their respective factors (appendix 3). For better interpretation of the model, the disability variable was removed from the model without changing the overall model fit and factor loadings.

Indices of fit, testing the structural model or the interrelations across factors, indicated that the proposed model fit the data moderately well $(\mathrm{CFI}=.892 ; \mathrm{CFI}$ corrected $=.57$; $\mathrm{RMSEA}=.06$; $90 \%$ confidence interval $[\mathrm{CI}], .055-.064)$. The model explained $50 \%$ of the variance of participation frequency and $51 \%$ of participation involvement. The correlation between the construct parameters as estimated by the structured means/full-information maximum likelihood model and the listwise deletion model was .986, which was accepted as evidence that the estimation method was not unduly influencing the results. Further investigation of the 
Table 1 PEM-CY scales and scores across the 3 settings: home, school, and community

\begin{tabular}{|c|c|c|c|}
\hline Variable & Scales & Scores & Range \\
\hline \multicolumn{4}{|l|}{ Participation } \\
\hline Frequency & 8 -point scale $(0=$ never to $7=$ daily $)$ & Mean of participation frequency* & $1-7$ (for each setting) \\
\hline Involvement & $\begin{array}{l}5 \text {-point scale }(1=\text { minimally involved to } \\
5=\text { very involved })\end{array}$ & Mean of participation involvement ${ }^{\dagger}$ & $1-5$ (for each setting) \\
\hline \multicolumn{4}{|l|}{ Environment } \\
\hline Barriers & $\begin{array}{l}\text { "Usually makes harder" or "no, usually } \\
\text { resource is not available/adequate" }\end{array}$ & $\begin{array}{l}\text { Mean of the number of items rated as } \\
\text { hindering/not available }\end{array}$ & $\begin{array}{l}\text { Home }(0-13) ; \text { school }(0-17) ; \\
\text { community }(0-16)\end{array}$ \\
\hline Supports & $\begin{array}{l}\text { "Usually helps" or "yes, usually resource } \\
\text { is available/adequate" }\end{array}$ & $\begin{array}{l}\text { Mean of the number of items rated as } \\
\text { helping/available }\end{array}$ & $\begin{array}{l}\text { Home }(0-13) ; \text { school }(0-17) ; \\
\text { community }(0-16)\end{array}$ \\
\hline
\end{tabular}

model yielded substantial support of its fit, as reported in appendix 2. As illustrated in figure 2, age and income were positively correlated with both aspects of home participation, and the number of health conditions (HCs) was negatively correlated with home participation. Environmental factors had a direct effect on home participation, where barriers decreased home participation (frequency and involvement) and supports increased home participation (frequency and involvement). Income, HCs, and functional issues also had an indirect effect on participation through their effect on environmental barriers and supports. In other words, both environmental barriers and supports served as mediators between personal factors (income), including the complexity of the child's condition (HCs, functional issues) and participation frequency and involvement. Specifically, higher income was associated with fewer barriers, whereas the number of $\mathrm{HCs}$ and the number of functional issues were associated with increased barriers, which in turn affected the levels of participation frequency and involvement.

\section{School model}

A total of 515 cases were available for analysis in the school model. The normalized estimate of multivariate kurtosis was 16.3, indicating a nonnormal distribution. All of the frequency and involvement items were significant indicators of their respective factors, and their standardized loadings were between .32 and .67 for the frequency variable and between .43 and .78 for involvement (see appendix 3). The proposed model fit the data well $(\mathrm{CFI}=.97$; CFI corrected $=.93$; $\mathrm{RMSEA}=.06 ; 90 \%$ CI, .051-.069) and explained 55\% of the variance of participation frequency and $61 \%$ of participation involvement. All exogenous variables related to complexity of the child's condition (functional issues, HCs, disability) and family income had a direct effect on participation involvement, whereas participation frequency was influenced only by functional issues and income (fig 3). Barriers, but not supports, had a direct effect on participation outcomes. Specifically, more HCs and functional issues were associated with more barriers, which in turn affected participation frequency and involvement.

\section{Community model}

There were 490 cases available for analysis in the community setting. The normalized estimate of multivariate kurtosis was 11.9; although lower than the previous models, it still indicates nonnormality. Similar to the school setting model, the age variable was not involved in any significant pathways and so was removed from the model. This resulted in a nearly identical model for the purpose of interpretation, but with an improved model fit for the data. The factorial structure of participation frequency and involvement was confirmed; all of the frequency and involvement items were significant indicators of their respective factors, and standardized loadings ranged between .20 and .72 for frequency and between .26 and .73 for involvement (see appendix 3). Disability did not function as a significant predictor in the model but was significantly associated with other demographic and complexity-related variables (HCs, functional issues).

The proposed model fit the data very well $(\mathrm{CFI}=.97$; CFI corrected $=.91 ; \quad$ RMSEA $=.042 ; \quad 90 \%$ CI, .036-.048) and explained $64 \%$ of the variance of participation frequency and $61 \%$ of participation involvement; both income and the number of functional issues had significant indirect effects on both the frequency and involvement of participation (fig 4). These indirect effects are due mostly to barriers (and to a small extent supports) serving as an intervening variable between income, functional issues, frequency and involvement in participation.

Table 2 Characteristics of subsamples in each model

\begin{tabular}{|c|c|c|c|c|c|c|c|c|c|}
\hline \multirow[b]{2}{*}{ Characteristic } & \multicolumn{3}{|c|}{ Home $(n=576)$} & \multicolumn{3}{|c|}{ School $(n=515)$} & \multicolumn{3}{|c|}{ Community $(n=490)$} \\
\hline & Mean & SD & Kurtosis & Mean & SD & Kurtosis & Mean & SD & Kurtosis \\
\hline Age $(y)$ & 11.15 & 3.08 & -1.057 & 11.15 & 3.11 & -1.08 & 11.18 & 3.07 & -1.08 \\
\hline No. of health conditions & 1.02 & 1.23 & -1.174 & 1.01 & 1.23 & -1.13 & 1.03 & 1.24 & -0.24 \\
\hline No. of functional issues & 3.13 & 3.78 & -1.191 & 3.08 & 3.78 & -1.16 & 3.17 & 3.80 & -1.23 \\
\hline
\end{tabular}




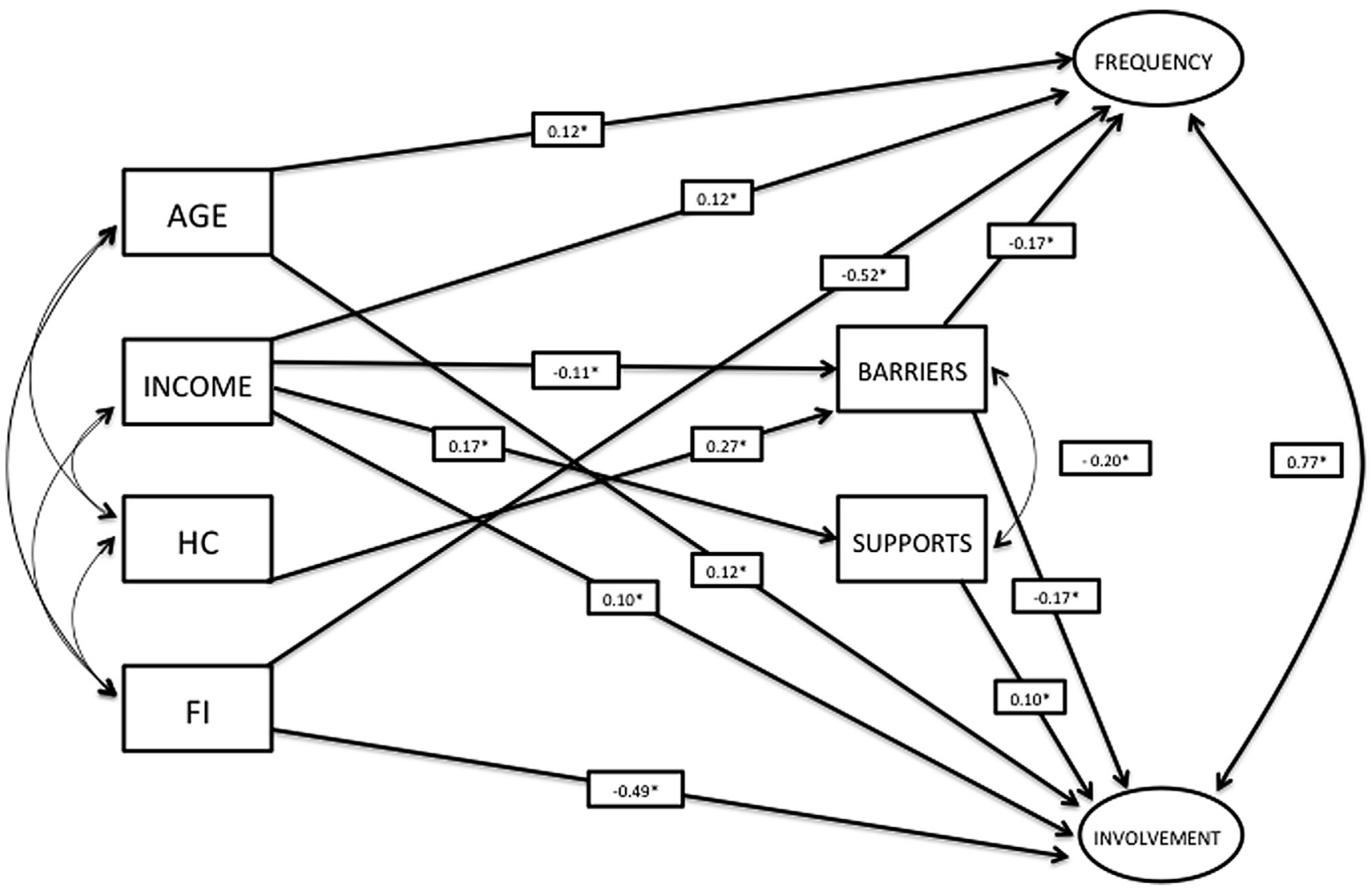

Fig 2 Structural model for explaining participation frequency and involvement in the home setting. For clarity, the indicators accounting for the latent variables of frequency and involvement were removed from the model and are presented in appendix 3 . FI, functional issue.

\section{Discussion}

This study contributes new knowledge about specific predictors of participation and their structural relations among children and youth in the home, school, and community settings. It specifically highlights the unique role of the environment as a mediator between child factors and participation. All three models, examined using robust conservative tests (ie, corrected CFI), fit the data well and accounted for $50 \%$ to $64 \%$ of the variance of participation outcomes-a considerably larger percentage in comparison with the results of previous models. For example, $18 \%$ to $30 \%$ of the variance of participation was explained in one study, ${ }^{24}$ and $14 \%$ to $30 \%$ was observed in another. ${ }^{18}$

\section{Role of child and family factors in explaining participation}

The demographic factors of income, as expected, had a direct and consistent effect on participation across all models. The effect of age, on the other hand, although known to influence participation in all children with and without disabilities, ${ }^{5,15}$ was negligible in the school and community settings. Previous studies ${ }^{5,14,15}$ indicated a shift in participation around the age of 12 years, with no evidence of cumulative changes. Furthermore, as opposed to previous studies, here age was modeled in the presence of dominant variables such as the complexity of the child's condition and environmental barriers, and consequently, its effect was less evident.
The complexity of the child's condition also played an interesting role. In the home model, the presence of a disability did not improve the model, and most of the participation variance was explained by age, income, functional issues, and number of health conditions. It is plausible that parents have more control within the home environment where routine is less formal. At the same time, the role of disability in the other models (ie, school and community) was minor. The mere presence or lack of a disability is not predictive, but rather the impact of the disability was seen through health conditions and functional issues.

The number of functional issues had a direct effect on participation, as well as an indirect effect through the environment, across all models. This further supports the findings of Law et $\mathrm{al}^{30}$ that the level of functioning is more important to participation than is the diagnosis, since one diagnosis does not necessarily capture the complexity of a child's condition. Similarly, Simeonsson et $\mathrm{al}^{31}$ discussed a dynamic view of disability in pediatrics, characterized by a shift from medical diagnosis to functional consequences of health conditions.

\section{Role of environment in explaining participation across settings}

While a comparison of the magnitude of effects or standardized coefficients across models is not statistically appropriate, descriptive trends across models were identified (fig 5). Environmental factors had a direct effect on participation across all settings; barriers consistently influenced both aspects of participation 


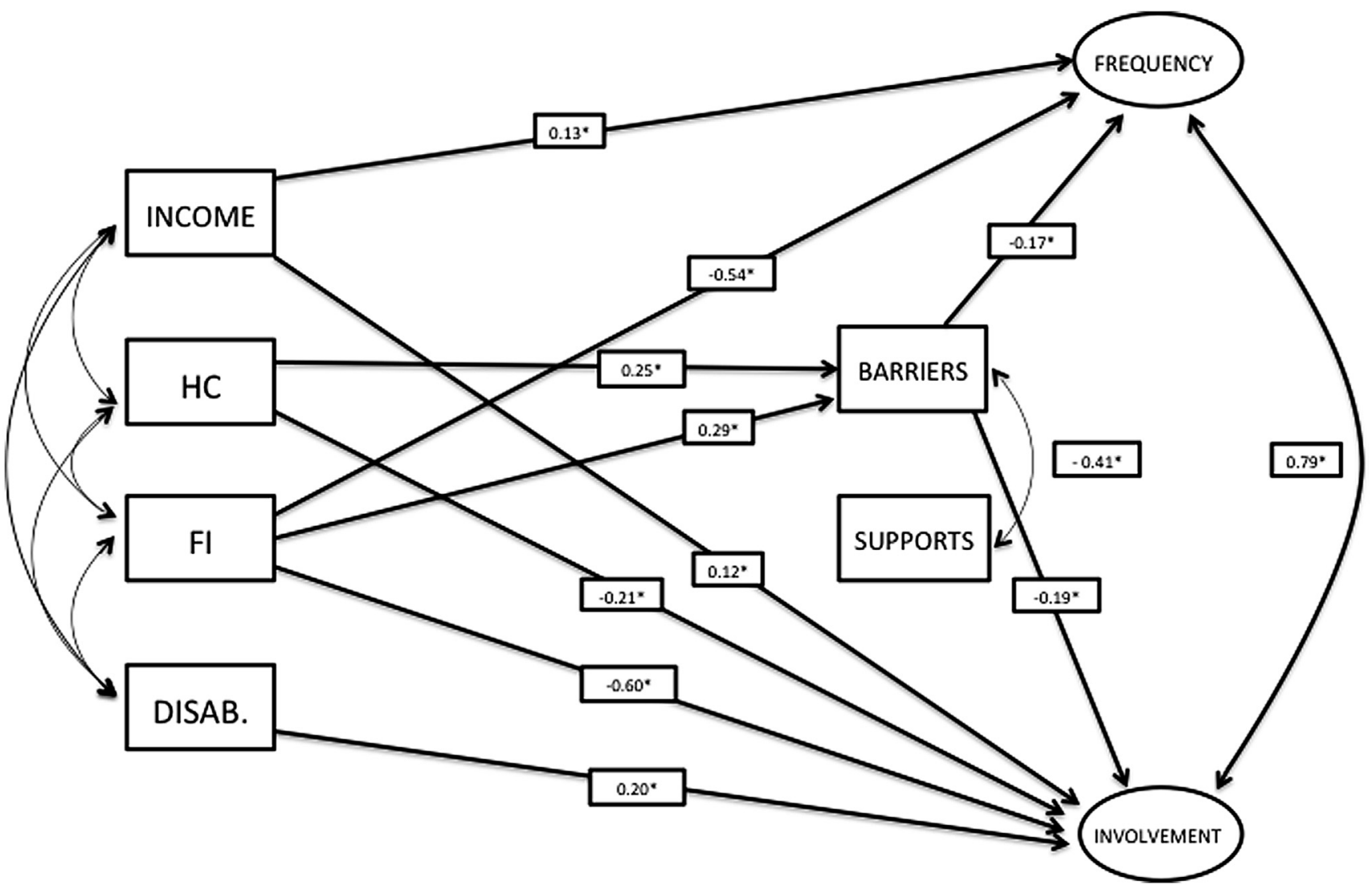

Fig 3 Structural model for explaining participation frequency and involvement in the school setting. For clarity, the indicators accounting for the latent variables of frequency and involvement were removed from the model. Abbreviation: DISAB., disability.

(ie, frequency and involvement), whereas the effect of supports was limited to specific settings such as home (only for involvement) and community. Overall, the effect of both environmental barriers and supports on participation was observed more frequently in the community setting. Environmental barriers also played an important role as an exclusive mediator, particularly in the community setting, meaning that without their presence, the effect of income and health conditions on participation would not have been observed.

While the direct impact of the environment was consistently observed across all settings in our study, this finding is incongruent with the model of King et al, ${ }^{24}$ where the environment had an indirect effect on participation. One reason may be how the environment was measured; $\mathrm{King}^{24}$ used the Craig Hospital Inventory of Environmental Factors, which measures general environmental barriers, whereas the PEM-CY directly links environmental features to participation, with greater specificity of the context of each setting: home, school, or community. Such an approach can capture explicit and more relevant aspects of the environment. Studies are needed to further explore these relationships.

Our findings also indicate that the relationship between the environment and participation differs across settings. The community setting, as opposed to the home and school, was the context in which the environment played a more substantial and unique role. Both barriers and supports consistently mediated the relationships between child factors and participation frequency and involvement. This may be due in part to the greater complexity of the community setting, which is in many cases harder to control. Common barriers in the community, found in the same sample studied here, included those related to the demand of the activity (ie, cognitive, physical, social), whereas supports involve the availability of resources such as programs, services, and information. ${ }^{9}$ Special attention can be given to potentially modifiable barriers and can examine whether changing environmental factors will impact participation. This finding is novel, as previous studies have simply measured the environment as a whole without considering the setting. It also stresses the utility of the PEM-CY, and consequently redirects attention to setting-based barriers and supports that can then be modified as leverage for inclusion.

\section{Clinical and research implications}

The finding that the environment served as a mediator between a child's factors and participation has clinical implications. The environment is more amenable to change compared with the child's health conditions and, in some cases, their functioning abilities. These findings direct therapists' attention toward intervention at the level of the environment and strengthen emerging context-based therapies ${ }^{32}$ that focus on changing the environment or the task, rather than the child's impairments, to improve function and participation. Pilot testing of a recently developed environment-based intervention to improve community participation $^{33}$ has also shown promise. It has become clear that the 


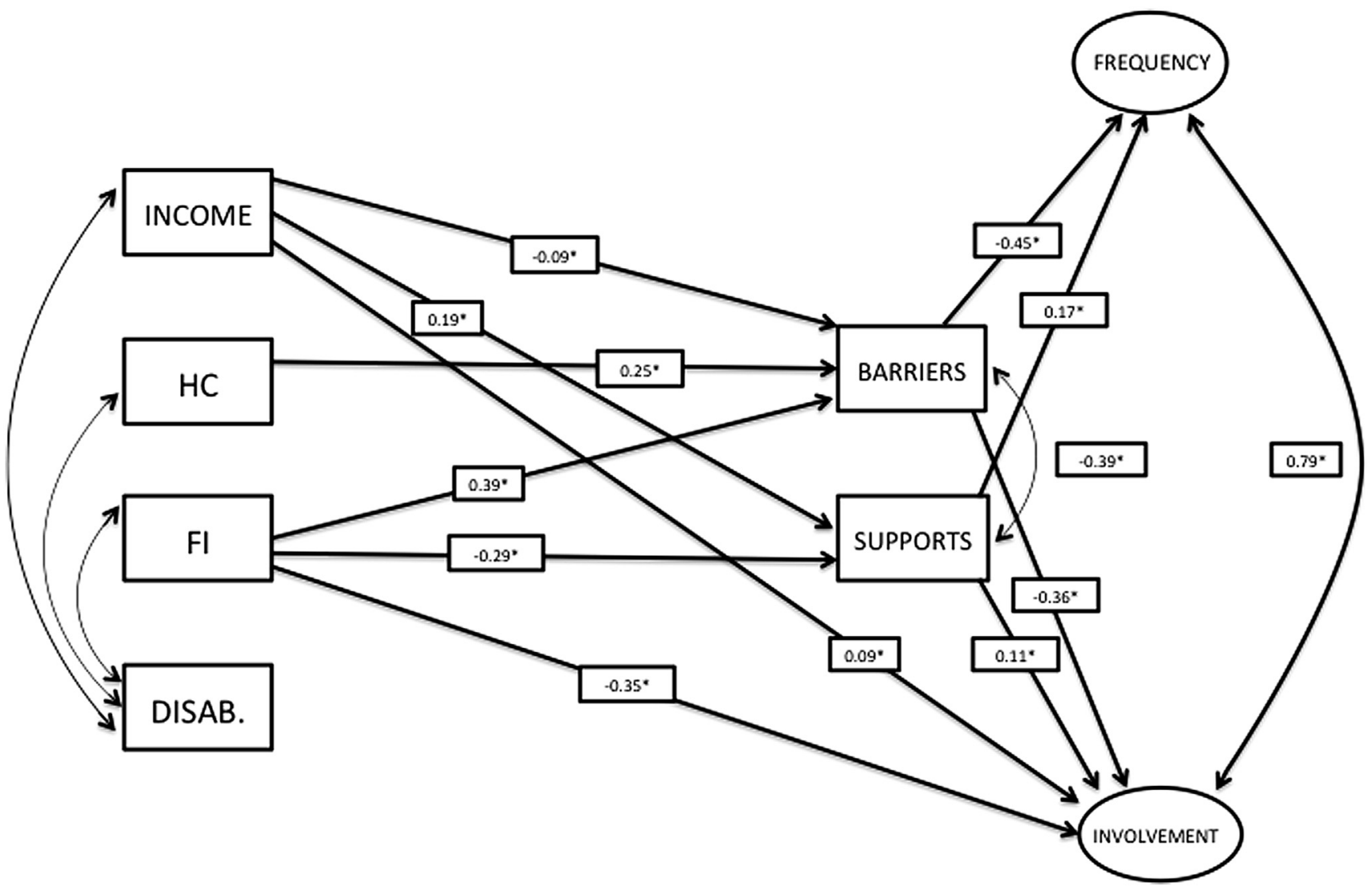

Fig 4 Structural model for explaining participation frequency and involvement in the community setting. For clarity, the indicators accounting for the latent variables of frequency and involvement were removed from the model. Abbreviation: DISAB., disability.

environment can serve as a target for intervention, and hence larger studies are needed.

Our findings also contribute evidence about the psychometric properties of the PEM-CY: the items of participation frequency and involvement accounted for their respective latent variables across all settings, supporting the construct validity of these concepts. Additionally, models behaved differently across settings, supporting the approach of the PEM-CY of evaluating each setting separately. Finally, our findings highlighted the critical role of the environment in explaining children's participation and may be of interest to other stakeholders such as policymakers of various organizations (eg, recreational, commercial, and community centers, religious institutions, schools). The interested groups can enact legislation to remove environmental barriers in order to promote inclusive communities.

\section{Study limitations and future directions}

This study examined the general impact of overall environmental barriers and supports. Investigation of the effects of specific environmental characteristics (eg, accessibility, attitudes), resources (eg, time, money, equipment), or activity demands (eg, physical, cognitive, social), which can be identified from the PEM-CY, is needed in the future. A child's desire for change in specific activities is another factor that can be generated from the PEM-CY; further models can include this factor to account for child/family preferences - an important determinant of participation. $^{24}$
The study included a convenience sample that may not represent the general population. Our sample, however, was heterogeneous with regard to types of diagnosis/health conditions represented. Testing the applicability of these models in diverse groups in terms of socioeconomic status and geographic locations is recommended.

Although SEM describes the direction and significance of paths, cause and effect cannot be claimed because of the crosssectional design of the study. Longitudinal intervention-based studies are needed for such inferences-for instance, observing change in participation after manipulation of the environment. Finally, while patterns of missing data were examined and robust methods were used, the common assumption that data are missing at random is not testable, and thus results should be interpreted with caution.

\section{Conclusions}

Our findings highlight the unique role of the environment in influencing participation across different settings and, thus, lend further support to developing and testing interventions that target the environment. This finding is of particular importance, since environmental barriers can be more practical to change in comparison with child functional issues - another important influence on participation. To complement our findings, further models can test the link between the environment and participation by teasing out the specific facets of the environment important to child's participation. 


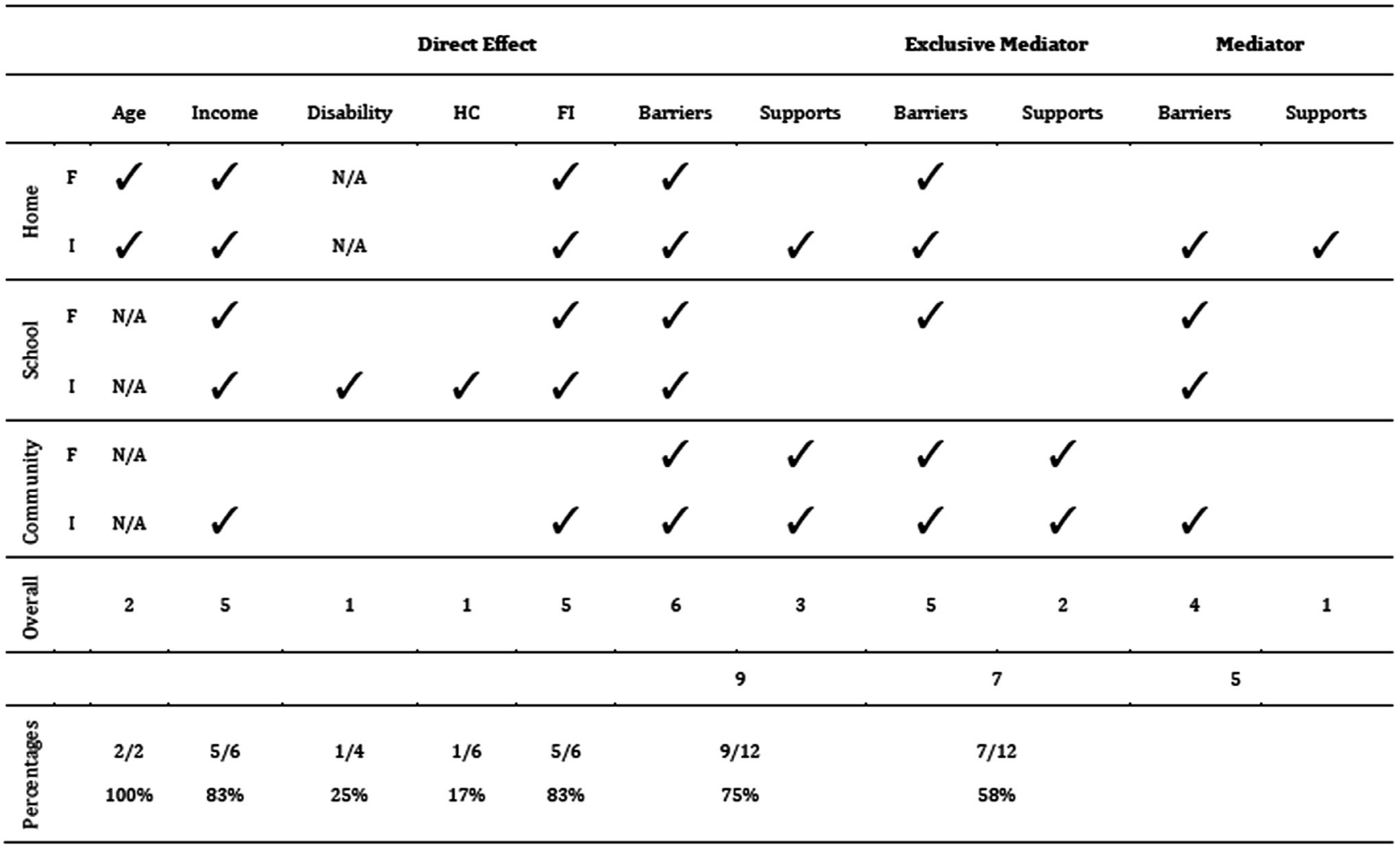

Fig 5 Summary of significant paths toward participation across models. Exclusive mediator: found in a situation where the effect of personal factors (eg, complexity of child's condition) on participation exists solely because of the presence of this mediator. Percentages represent the number of times an effect was observed, divided by the number possible options. Abbreviations: $F$, frequency; I, involvement; N/A, not applicable.

\section{Supplier}

a. Multivariate Software, Inc, 15720 Ventura Blvd, Ste 306, Encino, CA 91436-2989.

\section{Keywords}

Disabled children; Environment; Rehabilitation; Social participation

\section{Corresponding author}

Dana Anaby, PhD, 3630 Promenade Sir-William-Osler, Montreal, Quebec, Canada H3G 1Y5. E-mail address: dana.anaby@ mcgill.ca.

\section{Appendix 1 Recruitment Strategies}

Parents were recruited through the 3 participating universities situated in Ontario, Canada, and Massachusetts. Recruitment was facilitated by the cooperation of various organizations and family networks providing services for diverse children with disabilities and their families. Community-based agencies were also targeted to recruit children without disabilities-for example, sports and recreation centers and clubs in both regions. The recruitment assistance from these agency contacts was solicited in person by the research teams from each university at conferences, meetings, and presentations, as well as via e-mail and telephone. These agency contacts and staff were then provided with various recruitment materials such as pamphlets, flyers, and digital materials for distribution via organizations' newsletter, web posting, electronic mailing, and listserves to interested parents and their families. The research team did not contact parents directly. This recruitment process took place between May and October 2010.

\section{Appendix 2 Further Investigation of the Model Fit of Home Participation}

To further investigate the model fit, an identical model was run without the structured means and removing all cases with missing data (listwise deletion). This resulted in a model with identical uncorrected CFI and a CFI corrected for nonnormality of .868. The average SE was also similar at .474 , and the model Akaike Information Criteria - a measure of the relative quality of a statistical model-was higher at 274.138. When the construct parameters (the direct effects on the participation factors) were compared between the models, the correlation was very high at .986 . Such evidence further supports the claim that the model explaining participation in the home setting has a moderate fit with the data. 
Appendix 3 Factorial Structure of Participation Frequency and Involvement in the Home, School, and Community
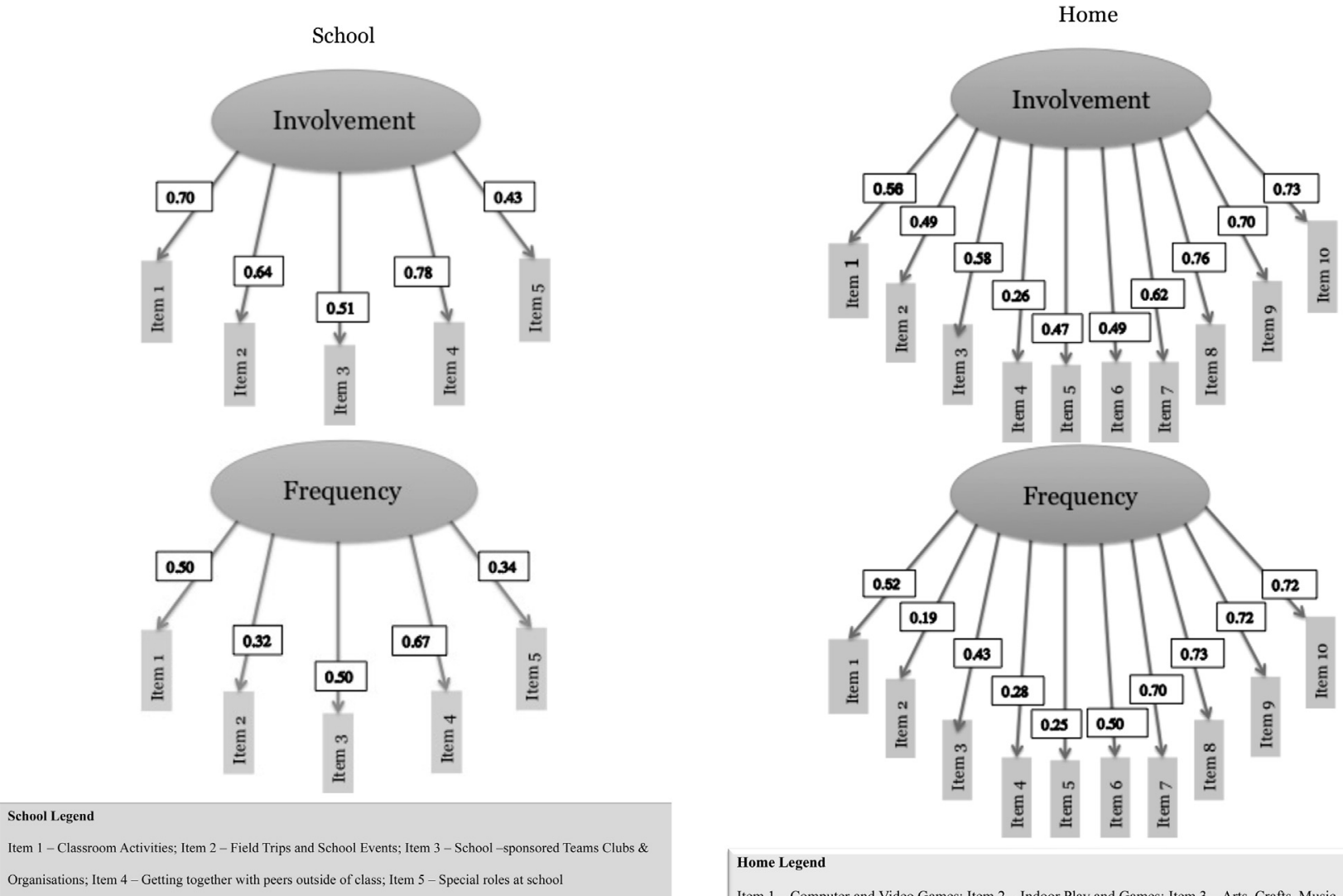

Organisations; Item 4 - Getting together with peers outside of class; Item 5 - Special roles at school

Home Legend

Item 1 - Computer and Video Games; Item 2 - Indoor Play and Games; Item 3 - Arts, Crafts, Music, and

Community

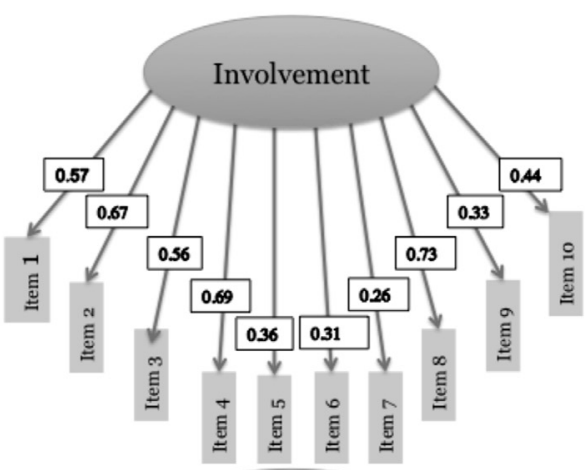

Hobbies; Item 4 - Watching TV, Videos and DVDs; Item 5 - Getting together with Other People; Item 6 -

Socializing Using Technologies; Item 7 - Household Chores; Item 8 - Personal Care Management; Item 9

School Preparation; Item 10 - Homework

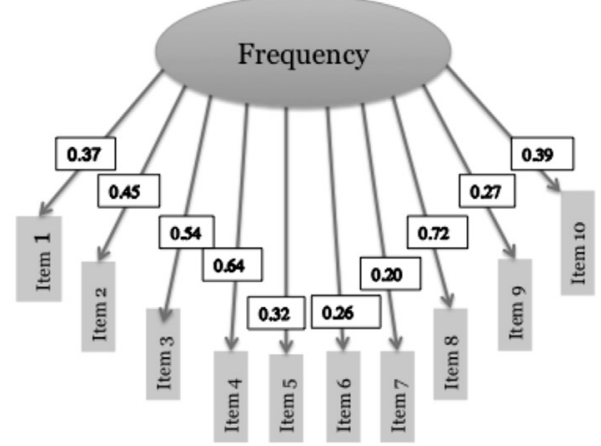

Community Legend

Item 1 - Neighbourhood outings; Item 2 - Community events; Item 3 - Organized Physical Activity; Item 4 -

Unstructured physical activity; Item 5 - classes and lessons; Item 6 - Organizations, groups clubs and volunteer or

leadership activities; Item 7 - Religious or spiritual gatherings and activities; Item 8 - Getting together with other children 


\section{References}

1. Larson RW. Toward a psychology of positive youth development. Am Psychol 2000;55:170-83.

2. Larson RW, Verma S. How children and adolescents spend time across the world: work, play, and developmental opportunities. Psychol Bull 1999;125:701-36.

3. Gorter JW, Stewart D, Woodbury-Smith M. Youth in transition: care, health and development. Child Care Health Dev 2011;37:757-63.

4. King G, Petrenchik T, Law M, Hurley P. The enjoyment of formal and informal recreation and leisure activities: a comparison of school-aged children with and without physical disabilities. Int J Disabil Dev Educ 2009;56:109-30.

5. King G, Law M, Hurley P, Petrenchik T, Schwellnus H. A developmental comparison of the out-of-school recreation and leisure activity participation of boys and girls with and without physical disabilities. Int J Disabil Dev Educ 2010;57:77-107.

6. Engel-Yeger B, Jarus T, Anaby D, Law M. Differences in patterns of participation between youths with cerebral palsy and typically developing peers. Am J Occup Ther 2009;63:96-104.

7. Majnemer A, Shevell M, Law M, et al. Participation and enjoyment of leisure activities in school-aged children with cerebral palsy. Dev Med Child Neurol 2008;50:751-8.

8. Law M, Anaby D, Dematteo C, Hanna S. Participation patterns of children with acquired brain injury. Brain Inj 2011;25:587-95.

9. Bedell G, Coster W, Law M, et al. Community participation, supports, and barriers of school-age children with and without disabilities. Arch Phys Med Rehabil 2013;94:315-23.

10. Michelsen SI, Flachs EM, Uldall P, et al. Frequency of participation of 8-12-year-old children with cerebral palsy: a multi-centre crosssectional European study. Eur J Paediatr Neurol 2009;13:165-77.

11. King G, Law M, King S, Rosenbaum P, Kertoy MK, Young NL. A conceptual model of the factors affecting the recreation and leisure participation of children with disabilities. Phys Occup Ther Pediatr 2003;23:63-90.

12. Hilton $\mathrm{CL}$, Crouch MC, Israel H. Out-of-school participation patterns in children with high-functioning autism spectrum disorders. Am J Occup Ther 2008;62:554-63.

13. Law M, King G, King S, et al. Patterns of participation in recreational and leisure activities among children with complex physical disabilities. Dev Med Child Neurol 2006;48:337-42.

14. Orlin MN, Palisano RJ, Chiarello LA, et al. Participation in home, extracurricular, and community activities among children and young people with cerebral palsy. Dev Med Child Neurol 2010;52:160-6.

15. Jarus T, Anaby D, Bart O, Engel-Yeger B, Law M. Childhood participation in after-school activities: what is to be expected? $\mathrm{Br} \mathrm{J}$ Occup Ther 2010;73:344-50.

16. Majnemer A, Shikako-Thomas K, Chokron N, et al. Leisure activity preferences for 6-to 12-year-old children with cerebral palsy. Dev Med Child Neurol 2010;52:167-73.

17. Anaby D, Law M, Hanna S, DeMatteo C. Predictors of chance in participation rates following acquired brain injury: results of a longitudinal study. Dev Med Child Neurol 2012;54:339-46.
18. King G, McDougall J, DeWit D, Petrenchik T, Hurley P, Law M. Predictors of change over time in the activity participation of children and youth with physical disabilities. Child Health Care 2009;38: 321-51.

19. Lawlor K, Mihaylov S, Welsh B, Jarvis S, Colver A. A qualitative study of the physical, social and attitudinal environments influencing the participation of children with cerebral palsy in northeast England. Pediatr Rehabil 2006;9:219-28.

20. Anaby D, Hand C, Bradley L, et al. The effect of the environment on participation of children and youth with disabilities: a scoping review. Disabil Rehabil 2013;35:1589-98.

21. Kramer J, Olsen S, Mermelstein M, Balcells A, Liljenquist K. Youth with disabilities' perspectives of the environment and participation: a qualitative meta-synthesis. Child Care Health Dev 2012;38:763-77.

22. Hammal D, Jarvis SN, Colver AF. Participation of children with cerebral palsy is influenced by where they live. Dev Med Child Neurol 2004;46:292-8.

23. Colver AF, Dickinson HO, Parkinson K, et al. Access of children with cerebral palsy to the physical, social and attitudinal environment they need: a cross-sectional European study. Disabil Rehabil 2011;33:28-35.

24. King G, Law M, Hanna S, et al. Predictors of the leisure and recreation participation of children with physical disabilities: a structural equation modeling analysis. Child Health Care 2006;35:209-34.

25. Coster W, Bedell G, Law M, et al. Psychometric evaluation of the Participation and Environment Measure for Children and Youth. Dev Med Child Neurol 2011;53:1030-7.

26. Law M, Anaby D, Teplicky R, Khetani MA, Coster W, Bedell G. Participation in the home environment among children and youth with and without disabilities. Br J Occup Ther 2013;76:58-66.

27. Coster W, Law M, Bedell G, Khetani M, Cousins M, Teplicky R. Development of the Participation and Environment Measure for Children and Youth: conceptual basis. Disabil Rehabil 2012;34: 238-46.

28. $\mathrm{Hu} \mathrm{L}-\mathrm{t}$, Bentler PM. Cutoff criteria for fit indexes in covariance structure analysis: conventional criteria versus new alternatives. Struct Equ Modeling 1999;6:1-55.

29. Bentler PM, Chou CP. Practical issues in structural modeling. Sociol Methods Res 1987;16:78-117.

30. Law M, Finkelman S, Hurley P, et al. Participation of children with physical disabilities: relationships with diagnosis, physical function, and demographic variables. Scand J Occup Ther 2004;11:156-62.

31. Simeonsson RJ, Leonardi M, Lollar D, Bjorck-Akesson E, Hollenweger J, Martinuzzi A. Applying the International Classification of Functioning, Disability and Health (ICF) to measure childhood disability. Disabil Rehabil 2003;25:602-10.

32. Darrah J, Law MC, Pollock N, et al. Context therapy: a new intervention approach for children with cerebral palsy. Dev Med Child Neurol 2011;53:615-20.

33. Law M, Anaby D, Turner L, Teplicky R, Imms C. Improving the participation of youth with physical disabilities in community occupations: an evaluation. Paper to be presented at: Meeting of the World Federation of Occupational Therapists; June 18-24, 2014; Yokohama (Japan). 